\title{
BIBLIOGRAFIA GERAL DO DIREITO ADMINISTRATIVO BRASILEIRO
}

- Catálogo coletivo das bibliotecas das seguintes instituiçōes : Departamento Administrativo do Serviço Público (DASP), Faculdade de Direito (FD), Serviço de Documentação do Ministério da Justiça (SDMJ), Ministério da Fazenda (MF), Supremo Tribunal Federal (STF), Tribunal de Apelação (TA), Tribunal de Contas (TC), Instituto de Apasentadoria e Pensóes dos Industriários (IAPI), Instituto de Apasentadoria e Pensões dos Empregados em Transportes e Cargas (IAPETC).

ALvEs, O Morais - Aposentadoria e pensóes dos ferroviários. Campinas S. T. F.

ANDrade, José Bonifácio Olinda de - A responsabilidado do estado em caso de guerra - D. A. S. P.

ANDRADE, Odilon C - Função públíca (natureza do vínculo-estatuto), Belo Horizonte, Imprensa Oficial do Estado de Minas, 1928, 137 p. D. A, S, P.

Serviços públicos e de utilidade pública, São Paulo, Saraiva 85 Cia, 1937, 149 p. - D. A. S. P., S. T. F. T T. C.

ARaújo CAstro - Estabilitade de funeionátos público, Rio do Janoiro, Leite Ribeiro e Maurilo, 1917, 143 p. - D. A. S. P., S. T. F. Q T. C.

AzEvedo, Washington - A organização técnica dos municípios, Rio de Janeiro, Pongeti, 1935,173 p. $-\mathrm{T} . \mathrm{C}$.

BANDEIRA DE MELo, Osvaldo Aranha - Aspectos da tegulamentação dos serviços públicos (In Direito, ano III, vol. XVII, setembro-outubro, 1942, ps. 94-119; vol. XVIII, novembro-dezembro, 1942, ps. 105-110) - D. A. S. P.

A executoriedade dos atos administrativos (in Direito, ano III, vol. XV, maio-junho, 1942, ps. 41-47) - D. A. S. P.

$A$ identidade de emprêsa nas serviços públicos (in Direito, ano $\mathbf{I}$, vol. XI, setembro-outubro, 1941 , ps. 58-75) _- D. A. S. P.

Municipalização dos serviços públicos, São Paulo, Revista dos Tribunais, 1939. 121 p. - D. A. S. P. e I. A. P. I.

NOTA DA RED.: O presente trabalho foi elaborado pela bibliotecária F. Marcondse Portugal, do Serviço de Referência da Biblioteca do D. A. S. P. 
Promoção, por sentença, São Paulo, Prefeitura do municipio do São Paúlo, 1943-44. 3 v., - D. A. S. P.

O tabelamento de gêneros pelos municipios.

Tributação de bens, rendas e serviças das unidades de tederaçäa Ballly, Gustavo Adolfo - Legislação social brasileira (sôbre funcionários públicos), Rio de Janeiro, 1933 - S. T. F.

Manual do funcionário público, Rio de Janeiro, A. Coelho Branco, 1938, 183 p. - S. T. F., D. A. S. P.

Seguro de estado, Rio de Janeiro, A. Coelho Branco, 1943, 438 p. D. A. S. P.

BIIAC PINTo - Regulamentação efetiva dos serviças de utilidade públion. Rio de Janeiro, Revista Forense, 1941,218 p. - S. T. F. T. C.

Bitroncourt, Carlos A. Lúcia - Imunidade fiscal das autarquias (in Revista do Serviço Público, ano V, vol. IV, n. 2, novembro de 1942 - Páginas 77-81). - Existe também em separata -D. A. S. P. - I. A. P. I.

A interpretação como parte integrante do processo legislativo (in Revista do Serviço Público, ano V, vol. IV, n.* 3, dezembro, 1942, Ps. 121-127). - D. A. S. P. I I. A. P. I.

O poder disciplinar e a lei penal cormum (in Revista do Serviço Público, ano V, vol. IV, n. ${ }^{\circ}$, outubro, 1942, p. 87-90). - D. A. S. P. e I. A. P. I.

A terminologia o o conceito de direito administrativo (in Revista do Serviço Público, ano VI, vol. III, n.o 2, a'gôsto, 1943, p. 75-79). D. A. S. P. I. A. P. I.

Branco, Plínio A. - Serviças de utilidade pública, São Paulo, Prefeitum municipal de São Paulo, 1941, 84 p. — T. C.

Brasu. (Departamento Administrativo do Serviço Público), Estatuto dow funcionários (estudos projetos), Rio de Janeiro, Imprensa Nacional, 1940, 418 p. - D. A. S. P.

Indicador da organização do executivo federal, Rio de Janeiro, Imprensa Nacional, 1944, 357 p. - D. A. S. P. e S. T. F.

Interpretação do estatuto das funcionários, 1939-41, Rio de Janeiro, Imprensa Nacional, 1942, 2 v. - D. A. S. P., S. D. M. J. I. A. P. I.

Jurisprudência administrativa (decisões e pareceres proferidos pelo D. A. S. P.), Rio de Janeiro, Imprensa Nacional, 1943, 5 v. D. A. S. P., S. D. M. J., I. A. P. I. e M. F.

Leis, decretos, etc., O Estatuto dos Funcionários Públicos Civis da União, Legislação posterior, Rio de Janeiro, Imprensa Nacional, 1942, 224 p. - D. A. S. P., S. D. M. J. e I. A. P. I.

Brisolla, Carlos Monteiro - Das lagos artificiais.

Campos, Francisco Luís da Silva - Direito administrativo, Rio de Janeiro, Imprensa Nacional, 1943, 359 p. - D. A. S. P., I. A. P. I., M. F., T. A. P T. C.

Pareceres, Rio de Janeiro, Tip. do "Jornal do Comércio", 1934, p. 71-293. - D. A. S. P.

Pareceres, 2." série, Rio de Janeiro, José Olímpio, 1936, p. 89-224.

- D. A. S. P. 
Campos Fllho, Paulo Barbosa de - Autarquias Federais o poder impositive local.

CARNEIRO, Alaim de Almeida. - Da retroatividade no direito administrative

(in Revista do Serviço Público, ano VI, v. IV, n. 1, outubro, 1943, p. 102-104). - D. A. S. P. e I. A. P. I.

Carnerro, Erimá - Autarquias (estudo do direito e finanças), Rio de Janeiro, Tip. do "Jomal do Comércio", 1937,90 p. - D. A. S. P.

As autarquias e as sociedades de economia mista no Estado Novo, Rio de Janeiro, Departamento de Imprensa e Propaganda, 1941. 222 p. - D. A. S. P., T. C. I. A. P. I.

Carnaro, Levi - Problemas municipais, Rio de Janeiro, 1931, 207 p. S. T. F. M. F.

CarvalHo, Frederico Cutio de - $O$ montepio civil; estudo sintético de ques. tôes relativas ao montepio civil, Rio de Janeiro, Imprensa Militar, $1922,342 \mathrm{p}$.

Carvalmo, Luís Antônio da Costa - Do direito da desapropriação, Rio do Janeiro, 1933. - S. T. F.

Carvalfo, Orlando M. - Problemas fundamentais do município, São Paulo, Editora Nacional, 1937, 174 p. (Brasiliana, n.* 84).

CARvalHo dE MENDonçA, Manuel Inácio - Rios a águas correntes em oua relaçóes jưrídicas, Rio do Janeiro, Freitas Bastos, 1939, 414 p. - Curitiba, 1909. - D. A. S. P. e S. T. F.

Carvalho a Melo, José Augusto - Prescrição (in Revista do Serviço Público, ano $V$, v. I, n.o 2 , fevereiro, 1942 , p. 36-39, n.o. 3, marco, 1942 , p. $33-37$; v. III, n. 1 , julho, 1942 , p. $71-76$; v. III, n. 2 , egôsto, p. 51-54) - D. A. S. P. e I. A. P. I.

Carvalho PINTo, Carlos Alberto - A cláusula-ouro nas concessōes de servigo público, São Paulo, Prefeitura Municipal de São Paulo, 1943, 112 p. - D. A.S. P.

Casasanta, Mário - Responsabilidade do estado por fatos de guerra, Belo Horizonte, $1932-$ S. T. F.

CASTRO NUNES, José de - Do estado feđerado e sua organização mumicipal. Histótia, doutrina, jurisprudência, direito comparado, Rio de Janeiro, Leite Ribeiro e Maurilo, 1920. 575 p. - I. A. P. I.

Cavalcantr, Amaro - Responsabilidade civil do estado, Rio de Janeiro, Laemmert, 1905. $634 \mathrm{p}$.

Cavalcantr, Augusto - A tabela Prado Júnior e os oficiais da Fazenda Mumicipal, Rio de Janeiro, 1931. - S. T. F.

Cavalcanti, Temistocles Brandão - Aleumas formas de autarquias mistas, territorial e institucional (in Revista do Servico Público, ano III, v. IV, n. 2, novembro, 1940, p. 65-68). - D. A. S. P. I. A. P. I. A codifícação do processo administrativo (in Direito, ano $I, \vee . I$, janeiro-fevereiro, 1940, p. 99-112). - D. A. S. P.

Código do processo administrativo. A unificação das normas do processo administrativo (in Revista do Serviço Público, ano I, v. III, n.o 2, agôsto, 1938, p. 54-68). - D. A. S. P. e I. A. P. I.

Conceito de administração (in Revista do Serviço Público, ano III, v. II, n. 3 , junho, 1940 , p. 83-85). D. A. S. P. I. A. P. I. 
Da hierarquia e do poder hierárquico no direito administrativo (in Revista do Serviço Público, año III, v. III, no i, julho, 1940 , p. 62-65). - D. A. S. P. I. A. P. I.

Da nulidade dos atos administrativos (in Revista do Serviço Público, ano V, v. I, n. 3, marco de 1942, p. 38-40) - D. A. S. P. e I. A. P. I.

Direito administrativo e ciência da administração (in Revista do Serviģo Público, ano III, v. I, n. 3 , março, 1940, p. 73-74). D. A. S. P. e I. A. P. I.

$O$ direito administrativo no Brasil (in Revista do Serviço Público, ano VII, v. .II, n. ${ }^{\circ}$, junho, 1944, p. 79-90, Direito, ano V, v. XXVI, março-abril, 1944, p. 32-53). - Existe, também, em separata. D. A. S. P. e I. A. P. I.

O direito administrativo, sua natureza, sua importancia, seus tratadistas (in Revista do Serviço Público, ano III, v. II, no 1, abril, 1940, p. 68-70) - D. A. S. P. e I. A. P. I.

Direito disciplinar (in Revista do Serviço Público, ano III, v. II, n. 2 , maio, 1940, p. 130-132). - D. A. S. P. e I. A. P. I.

Empréstimos públicos; suspensão do pagamento e seus efeitos jurídicos (in Direito, ano I, v. III, maio-junho, 1940, p. 52-65)

D. A. S. P. I. A. P. I.

O estado, a administração e os serviços públicos no mưndo democrático, conferência feita na Academia Brasileira de Letras, promovida pelo P. E. N., em 29 de setembro de 1943 (in Revista do Serviço Público, ano VI, v. IV, n. ${ }^{\circ}$, novembro, 1943, p. 12-15). D. A. S. P. I I. A. P. I.

A função pública e seu regime juridico (in Revista do Serviço Público, ano I, vol. I, n. 4 , março de 1938, p. 35-43; v. II, n.o 1 , abril, p. 43-51; n.o 2, maio, p. $30-38$; n. 3 , junho, p. 42-49; v. III, n. ${ }^{\circ} 1$, julho, p. $38-55$; n. 3 , setembNo, p. 31-38; v. IV, n. .1 , outubro, p. $62-71$; n.o 2 , novembro, p. 78-85; ano II, v. I, n. 1 , janeiro, 1939 , p. $35-40$; ns. 2 e 3 , fevereiro e março, p. $39-47$; v. II, ns. 1 e 2 , abril e maio, p. 76-82; no 3 , junho, p. 71-76; v. III, ns. 1 e 2, julho e agôsto, p. 45-50; n. 3, setembro, p. 38-42; ano III, v. I, n. 1 , janeiro, 1940, p. 52-56). - D. A. S. P. I. A. P. I.

O funcionário público e o seu estatuto, Rio de Janeiro, Freitas Bastos, 1940, 490 p. - D. A. S. P., M. F. e T. C.

Instituiçóes de direito administrativo brasileiro, Rio de Janeiro, Freitas Bastos, $1936-2^{2}$ ed., 1938, 2 v. - D. A. S. P., S. T. F., T. C., T. A., I, A. P. I e I, A. P. E. T C.

O Instituto de Resseguros do Brasil como entidade autárquica (in Revista do Serviço Público, ano IV, v. I, n. ${ }^{\circ}$, janeiro, 1941, p. 49-54). - D. A. S. P. I. A. P. I.

Órgãas e departamentos autônomos (in Revista do Serviço Público, ano IV, v. IV, n. 1 , outubro, 1941 , p. 63-65). - D. A. S. P. I. A. P. I.

o princípio de retroatividade e o direito público (in Revista do Serviço Público, ano V, v. I, n. 2, fevereiro, 1942, p. 64-66). D. A. S. P. I. A. P. I. 
Serviços de utilidade pública (Revista do Serviço Público, ano IV, V. I, n.o 3, março, 1941, p. 69-73) - D A. S. P. e I. A. P. I.

Serviços de utilidade pública e sua regulamentação (in Direito ano IV, V. XIX, janeiro-fevereiro, 1943 p. 14-24). - D.A.S. P.

Sôbre a intervenção do estado (in Revista do Serviço Público, ano IV, v. III, n. ${ }^{\circ}$, agôsto, 1941, p. 85-89) - D. A. S. P. e I. A. P. I.

Tavares Bastas (A liberdade pela descentralização) (in Revista do Brasil, agôsto, 1939).

As tendências modernas do direito administrativo (in Revista do Serviço Público, ano VI, v. I, no 2 , fevereiro, 1942, p. 111-119, Direito, ano III, v. XVIII, novembro-dezembro, 1942, p. 18-36) . D. A. S. P. I. A. P. I.

A teoria do silêncio no direito administrativo (In Revista do Serviço Público, ano III, v. I, n. 2, Fevereiro, 1940, p. 4548, Revista da Faculdade de Direito de São Paulo, v. XXXIV, fasc. II, maioagôsto, 1938, p. 122-130) - D. A. S. P. e I. A. P. I.

Tratado de direito administrativo, Rio de Janeiro, Freitas Bastos, 1942. 6 v. - D. A. S. P., S. D. M. J., M. F., F. D., T. A., T. C. e I. A. P E, T, C.

Tribunais administrativos e contrôle judiciário dos atos administrativo (in Revista do Servico Público ano IV, $v, \mathrm{II}, \mathrm{n} .^{\circ} 1$, abrit 1941, p. 206-209) - D. A. S. P. e I. A. P. I.

O tribunal administrativo e a apreciação judiciátia dos atos administrativos (in Revista do Serviço Público, ano IV, v. II, n.॰ 3, junho, 1941, p. 100-104) - D. A. S. P.

Uma experiência administrativa; as colônias agrícolas (in Revista do Serviço Público, ano, IV, v. II, n. 2, maio, 1941, p. 90-93). D. A. S. P e I. A P I.

Chaves Luís de Sousa Leite - Exercício da funçáo pública e direito aos vencimentos.

Comlho, Henrique - A vitaliciedade dos empregados públicos, São Paulo, Vanorden \& Cia., 1908, 16 p. - D. A. S. P.

CoRreia, Luís M. O estado e a obrigação de indenizar, Rio de Janeiro, 1920. - S T. F.

Costa, Duque - Das concessóes administrativas de privilégios, Belo Horizonte, Imprensa oficial de Minas Gerais, 1930, $61 \mathrm{p}$.

CRUz, Alcides - Direito administrativo brasileiro, 2.a ed. corrigida e ampliada, Rio de Janeiro, Francisco Alves 86 Cia., 1914, 264 p. - D. A. S. P. T. C. I. A. P. I.

Cunha Mexo, Leopoldo - Responsabilidade da Fazenda nacional pelos atos do interventor no Amazonas, Rio de Janeiro, 1928. - S. T. F.

DELGADo, Luís - Autarquias : função social e aspectos jứídicos, Recife, 1940.

De Plácmo e SHLVA, Oscar José - As caixas econômicas federais, sua história, sua administração e operações autorizadas, Curitiba, Graf. Paranaense, 1937,519 p. - D. A. S. P. e.M. F.

Drumond Alves, Eduardo de - Promoção e seu regulamento, Rio de Janeiro, 1944, 185 p. - D. A. S. P. e S. D. M. J. 
Fmrrelra, Silvestre Pinheiro - Indicaçäo de utïidado puiblica, Paris, 1834. - S. T. F.

Flerus, MAx - Histótia administrativa do Brasil, Rio de Janeiro, Imprense Nacional, 1923, 358 p. - 2." ed., São Paulo, Companhia Melhoramentos de São Paulo, 844 p. - D. A. S. P. S. D. M. J.

Fonspec, Tito Prates da - Atos administrativos nulos e anuláveis - (in Direito, ano III, v. XIII, janeiro-fevereiro, 1942, p. 45-69). D. A. S. P.

- Autauquias administrativas, São Paulo, Savaiva \& Cia, 1935. 15 p. - D.A.S.P., S.D.M.J., S.T.F.T.C. I.A.P.E.T.C $A$ causa em direito administrativo (in Direito, ano III, จ. XIV março-abril, 1942, p. 31-51). - D. A. S. P.

Direito administrativo, Rio de Janeiro, Freitas Bastos, 1939, 439 p. D. A. S. P., T. C. I. A. P. E. T. C.

A evolução do direito administrativo brasileiro nos últimos 25 ano (in Direito, ano III, v. XVII, setembro-outubro, 1942, p. 23-37). D. A. S. P.

- Lições de direito administrativo, Rio de Janeiro, Freitas Bastos, 1943, 431 p. - D. A. S. P. T. C.

FORTE, Horácio S. - Das fraudes aduaneiras; processo administrativo, Recife, Of. Graf. do Jornal do Comércio, 1940, 468 p. — D. A. S. P.

Franoo Sobrinho, Manuel Oliveira - Autarquias administrativas, São Paulo, Revista das Tribunais, 1939, 180 p. - D. A. S. P., S. D. M. J. T. C.

Avaliação de bens e capital para efeito de desapropriação das entidades delegadas de serviço público (in Direito, ano II, v. IX, maio-junho, 1941, p. 101-112). - D. A. S. P.

Concessão de serviços públicos em direito edministrativo. - Teeo apresentada ao I Congresso Universitário do Brasil, reunido en Salvador, Curitiba, Tip. Joäo Haupt \& Cia., 1936, 27 p.

Da posição juridica do direito processual administrativo (in Direito, ano I, v. II, março-abril, 1940, p. 88-101). - D. A. S. P.

Desapropriação por utilidade pública, 1942.

Do conceito do contrato administrativo.

Os serviços de utilidade pública, 1940.

Garcia, Basileu - Dos crimes contra a administraçäo pública (in Revista do Serviço Público, ano III, v. IV, n. 1, outubro, 1944, p. 93-103; n. 3, dezembro, p. 98-104). - D. A. S. P. e I. A. P. I.

Gomes, José Bezerra - Em tôrno das terras devolutas (in Direito, ano III, v. XIII, janeiro-fevereiro, 1942, p. 111-116). - D .A. S. P.

Junquerra, Messias - Terras devolutas (in Direito, ano II, v. IX, maiojunho, 1941, p. 153-178). - D. A. S. P.

Leal, Aurelino d'Araújo - Polícia e poder de polícia, Rio de Janeiro, Imprensa Nacional, 1918,316 p. - S. T. F.

LEIre, Solidônio - Desapropriação por utilidade pública, Rio de Janeiro, Imprensa Gutemberg, 1903, $160 \mathrm{p}$.

2. od. 1921. - S. T. F. \& T. C. 
Lran, Adamastor - As emprêsas elétricas e a restituiçăo das quăintias quo, aos consumidores, forem cobradas a mais (in Direito, ano IV, v. XXIV, novembro-dezembro, 1943, p. 67-85). - D. A. S. P.

O Estado, os servidores públicos, as emprêsas concessionárias e o capital respectivo (in Direito, ano III, v. XIV, março-abril, 1942, p. 68-79). - D. A. S. P.

LIMA, Hermes - Atividades administrativas do estado. - Tese para concurso, São Paulo, Saraiva \& Cia., 1927, 57 p.). - T. C.

LIMA, Rui Cirne - Introdução ao estudo do direito administrativo brasileiro, Pôrto Alegre, Livraria do Globo, 1942, 138 p. - D. A. S. P. T. C.

Princípios de direito administrativo brasileiro, Pôrto Alegre, Livraria do Globo, 1939, 216 p. - D. A. S. P., T. C. I. A. P. E. T. C. Terras devolutas, Pôrto Alegre, 1935. - S. T. F.

LIRA, Paulo - Tendências do direito administrativo brasileiro no Estado Novo (in Revista do Serviço Público, ano IV, v. II, n. 3 , junho, 1941, p. 116-124). - Existe também em separata. - D. A. S. P. I. A. P. I.

LIRA, Roberto - Crimes contra a administração pública (in Revista do Serviço Público, ano VII, v. I, n.o 1, janeiro, 1944, p. 107-110; n. 2 , fevereiro, p. 105-107; v. II, n. 1 , abril, p. 96-98; n. 2 , maio, p. 119-122; v. III, n. 1 , julho, p. 124-126; n.o 3 , setembro, p. 117-119; v. IV, n.o 2, novembro, p. 96-98). - D. A. S. P. I. A. P. I.

Lopes, Américo Ferreira - Administração municipal, Rio de Janeiro, 1935. - S. T. F.

MACHADo NETo, João Gonçalves - Questós administrativas e fiscais, Rio de Janeiro, Of. d'“A Balança", 1934, 143 p. - M. F.

MadRUGa, Manuel - Terrenos de marinha, Rio de Janeiro, Imprensa Nacional, 1928, 2 v. - S. T. F.

Mata, João Azevedo de Carneiro - $O$ município, 1863.

Masagão, Mário - Gonceito do direito administrativo, São Paulo, 1926. Em face da constituição federal náo existe no Brasil o contencioso administrativo, São Paulo, 1927.

Natureza jurídica da concessão de serviço público, São Paulo, Saraiva \& Cia., 114 p. - D. A. S. P., S. T. F., F. D., T. C., F. D., I. A. P. I. I. A. P. E. T. C.

Mederros, Océlio - Administração e legislação de fronteitas (in Revista do Serviço Público, ano VII, v. III, n..$^{\circ}$, setembro, 1944, p. 30-41). - D. A. S. P. e I. A. P. I.

MERA, Otávio - Do direito de desapropriar, Pará, 1934.

Merra E SÁ, F. de S. - $O$ direito invertido. Sôbre a proposição da Câmara dos Deputados referente à aposentadoria dos funcionários públicos, Natal, Tipografia do Instituto, 1914,98 p. - D. A. S. P.

MELo, Luís Anhaia - $O$ problema econômico dos serviços do utilidado pública (Palestras realizadas no Instituto de Engenharia), São Paulo, Escolas Profissionais do Liceu Coração de Jesus, 1930, 188 p. 
MENDonf̧, Francisco Matia Furtaủo de - Excerto de aireito administrativo brasileiro, 1865.

Menegale, J. Guimarães - Direito administrativo e ciência da administração, Rio de Janeiro, Metrópole editốra, 1938, 3 v. - D. A. S. P., S. T. F., T. C. $\mathbf{M}$. F.

MENEZES, Djacir de - Direito administrativo moderno, Rio de Janeiro, Coelho Branco, 1943, 275 p. - D. A. S. P.

MONIz SODRÉ - Estatuto dos funcronários públicos, Rio de Janeiro, Imprensa Nacional, 1913, 87 p. - D. A. S. P. e S. T. F.

MONTEIRo DE RESENDE, Lisandro - Da consignação em fôlhas; história, doun trina, legislação e jurisprudência, Rio de Janeiro, 1941,310 p. D. A. S. P.

Moreno, S. F. - O caso dos bondes.

Direito público subjetivo, São Paulo, Departamento Jurídico da Prefeitura de São Paulo, 1940, 97 p. — D. A. S. P.

Munhoz, Laertes - Da perda da tunção pública como pena acessória (in Revista do Serviço Público, ano VI, v. III, n.o 3, setembro, 1943, p. 82-84). - D. A. S. P. e I. A. P. I.

Nunes, Pedro Severiano - Da morfodinâmica do municipio (Tese de concurso) Manaus, 1940.

OLIVEIRA, A. Gonçalves de - Responsabilidade civil do estado por ato legis. lativo - cláusula ouro

Olrverra, José Rubino de - Epítome de direito administrativo brasileiro, São Paulo, 1884.

OLIverRa SANTos - Direito administrativo e ciência da administração, Rio de Janeiro, Jacinto Ribeiro dos Santos, 1919, 412 p. - D. A. S. P., S. T. F., F D. E T. C.

OLIVELRA E SILVA - O munić́pio no Estado Novo (Doutrina, jurisprudência e legislação sôbre o município e o seu funcionário), Rio de Janeiro, 1940, 311 p. - D. A. S. P., T. C. F F. D.

Ouro Prêto, Afonso Celso de Assis Figueiredo - Reforma administrativa e municipal; parecer e projetos, Rio de Janeiro, Imprensa Nacional, 1883. - S. T. F.

OURo PRÊTo, Luís V. Belfort de - Dos deveres e da ação disciplinar (in Revista do Serviço Público), ano VII, v. III, no 2, agôsto, 1944, p. $48-52$; n. 3 , setembro, p. 81-89; v. IV, no 1, outubro, p. 55-59; n. 3, dezembro, p. $61-63$ - D. A. S. P. I I. A. P. I.

PaAshaus, Gustavo Cintra - Das causaçôes de funcionários, Recife, 1943, 73 p. - D. A. S. P.

Parva, Antônio Vasconcelos de - Notas sôbre terrenos de marinhas, Paraíba, Imprensa oficial, 1925, 154 p. - D. A. S. P.

PANDiÁ Calógeras, João - Problemas de administração, $2 .^{a}$ ed, São Paulo, Editôra Nacional, 1938, 274 p. Brasiliana, v. 24. D. A. S. P. e M. $\mathbf{F}$.

Pelxoro, Moacir Matos - Autarquias (in Revista do Serviço Público, ano VII, v. 1 , n. 2 , fevereiro, 1944 , p. $39-42$. D. A. S. P. I. A. P. I. 
PENA, Fernando - Contrôle jưriślicional, Belo Horizonte, Oliveira Costa \& Cia., 1933, 183 p.

Pereira, Andrá de Faria - Contratos de serviços de utilidade pública (in Direito, ano II, v. X, julho-agôsto, 1941, p. 164-166, — D. A. S. P. e S. D. M. J.

PEReira da Silva, Luciano - Questóes jurídicas em processos administrativos, Rio de Janeiro, Serviço de Informação Agrícola, 1942-44, 4 v. D. A. S. P. A I. A. P, E. T. C.

Pessoa, Eduardo Pinto - Estatuto dos funcionários públicos civis da União e leis correlatas, Rio de Janeiro, Impressora di Pauli Ltda., 1940, 281 p. - D. A. S. P. e T. C.

PessoA, Epitácio - Terrenos de marinha, Rio de Janeiro, 1904_- S, T, F. Manual dos servidores do estado, Rio de Janeiro, 1943,2 v. D. A. S. P. e S. D. M. J.

Pinhemo, Alcides - Direito das minas; comentários a legislação, Rio de Janeiro, Jornal do Comércio, 1939, 222 p. - T. C.

Pôrto Carreiro, Carlos - Lições de direito administrativo, Rio de Janeiro, 1918. - S. T. F.

Prazeres, Oto - Os Estados poderáo cobrar selos judiciátios (in Revista do Serviço Público, ano V; v. I, n. 1 , janeiro, 1942, p. 149-152). D. A. S. P. E I. A. P. I.

QUENTEL, Oscar Sampaio - Do processo administrativo (in Revista do Serviço Público, ano V, v. I, n.o 1, Janeiro, 1942, p. 73-76; n. 2, fevereiro, p. $67-70$; v. II, n. 1 , abril, p. 35-37; n. 3 , junho, 1942, p. 65-67). - D. A. S. P. A I. A. P.I.

REs, Aaráo - Direito administrativo brasileiro, Rio de Janeiro, Of, Gráfica Vilas Boas, $1923,639 \mathrm{p},-\mathrm{S}, \mathrm{T}, \mathrm{F}, \mathrm{F}, \mathrm{D}, \mathrm{T}, \mathrm{C}$. e T. A.

RêGo, Vicente Pereira do - Compéndio ou repetiçóes escritas sóbre os elementos de direito administrativo para uso das faculdades de direito do Império, Recife, D. F. das Neves Guimarães, 1877,412 p. - F. D.

RÊGo BAkros, Fentque - Apontamento sobre o contencroso administrativo, Rio de Janeiro, Eduardo e Henrique Laemmert, 1874,646 p. S. T. F e T. C.

RESEnde, Jair - Terrenos de marinha (Manual prático) Rio de Janeiro, Of. Graf, I. Amorim Ltda, 1937, 179 p. - D. A. S. P. e S. T.F.

Resende Tostes, Lair Paleta - Coletânea de opiniōes, artigos o memórias sôbre os serviços de utilidade pública, Rio de Janeiro, 1942, 204 p. D. A. S. P.

Serviços de utilidade pública e sua base de tarifa, Rio de Janeiro, Freitas Bastos, 1940, 188 p. - 1941, 119 p. - D. A. S. P., S. D. M. J. I I. A. P. E. T. C.

RiBas, Antônio Joaquim - Direito administrativo brasileiro, Rio de Janeiro, F. L. Pinto \&6 Cia, 1866,507 p. - Obra premiada e aprovada pela resolução imperial de 9-2-1861 para uso das faculdades de direito de Recife e São Paulo. - I. A. P. I., S. D., T. C., S. T. F. e.M. F.

Rocha, Artur - Da intervenção do Estado nos contratos concluídos, Rio de Janeiro, 1932. - S. T. F. 
Roppico Otávio Langgaard de Meneses - Do domínio da Uniăo o das Estados, segundo a constituiçāo federal, Rio de Janeiro, Imprense Nacional, 1897, 108 p.

2. ad. São Paulo, Saraiva \& Cia., 1924, 239 p. - M. F. (ed. 1897) e D. A. S. P. (ed. 1924).

Rodrigues Vale, José - Curso de direito administrativo, Rio de Janeiro, A. Coelho Branco, 1941, 380 p. - D. A. S. P., S. D. M. J., S. T. F., F. D., M. F., T. C. $\theta$ T. A.

Rosa, Rubem - Direito e administração, Rio de Janeiro, Jornal do Comércio, 1940, 348 p. - D. A. S. P., S. D. M. J. O T. C.

Salasar, Alcino de Paula - Concessöes de serviços públicos no direito brast. leiro atual (in Revista de Jurispruxência Brasileira, V. XIII, Janeiro, 1939, p. 249-261. - D. A. S. P.

Responsabilidade do poder público por atos judiciais.

Santos, Moacir Amaral - Responsabilidades dos proprietários em face dos regulamentos administrativos sóbre o direito de construir.

São Pauro, Prefeitura do município de - Departamento Jurídico - Serviço telefônico do municipio da capital - Documentação relativa à concessão vigente, São Paulo, 1941, 301 p. - D. A. S. P.

SARAIVA, Oscar - Novas formas da delegação administrativa do ostado; as sociedades de economia mista e as fundações públicas (in Revista do Serviço Público, ano VII, v. ILI, n. ${ }^{\circ}$, setembro, 1944, p. 114-117). - D. A. S. P. I. A. P. I.

Organização da administração delegada, Rio de Janeiro, Serviço de Documentação do D. A. S. P., 1942, 12 p. - D. A. S. P.

Seabra Fagundes, Miguel - $O$ contrôle dos atos administrativos pelo poder judiciário, Rio de Janeiro, Freitas Bastos, 1941, 446 p.) - D. A. S. P., S. D. M. J., T. C. S. T. F.

Da desapropriação no direito brasileiro, Rio de Janeiro, Freitas Bastos, 1942, 506 p. - T. C.

Dos terceiros em face da desapropriação (in Direito, ano III, v. XVII, setembro-outubro, 1942, p. 68-80). - D. A. S. P.

Da proteção do indivíduo contra o ato administrativo ilegal ou abusivo (in Revista do Serviço Público, novembro, 1943, p. 87-106). - D. A. S. P. e I. A. P. I.

Stgadas Viana, José de - Os extranumerários das emprêsas de serviças públicos administrados pela União e a justiça do trabalho (in Direito, ano III, v. XV, maio-junho, 1942, p. 7-24). - D. A. S. P.

Serpa, José de - Estudos de direito administrativo, Rio de Janeiro, Casa Gráfica, 1927, 174 p. - D. A. S. P., S. T. F. e T. C.

SprNola, Celso - Desapropriação por necessidade ou utilidade pública, Rio de Janeiro, J. Ribeiro dos Santos, 1930, 357 p.

Jacinto editor, 1941,441 p. - S. T. F. e T. C.

SODRÉ, Eurico - A desapropriação por necessidade ou utilidade pública, São Paulo, Saraiva \& Cia., 1928, 231 p. - S. T. F.

SousA, Amâncio de - Responsabilidade funcional dos secretátios de Estado, Bahia, 1906. - S. T. F. 
Sousa BANDEIRA, J. C. - Proleçóes de ciência da administraçao e direito administrativo do Dr. J. C. de Sousa Bandeira, resumidas e organizadas por Vicente de Sabóia Lima e Melo Barreto Filho, Rio de Janeiro, Of. Gráf., 1913, 151 p. - D. A. S. P.

Tavares Bastos, Aureliano Cândido - Cartas do solitário, $3 .^{a}$ ed. feita sôbre a 2. ed. de 1863, São Paulo, Editôta Nacional, 1938, 521 p. (Brasiliana, v. 115). - D. A. S. P.

A província, estudo sâbre a descentralização no Brasil, $2 .^{a}$ ed. feita sôbre a 1." ed. de 1870, São Paulo, Editôra Nacional, 1937, 383 p. (Brasiliana, v. 105) - D. A. S. P.

TAvares DE LIRA, Augusto - Contratos administrativa, Rio de Janeiro, Of. Gráf. do Jornal do Brasil, 1941, 190 p. - D. A. S. P., T. C. e I. A. P. E. T. C.

Organização política e administrativa do Brasil (Colônia, Império e República), São Paulo, Editôra Nacional, 1941 (Brasiliana, v. 202). - M. F.

Trxxerra, J. H. Meireles - O problema das tarifas nos serviços públicas concedidos, São Paulo, Departamento Jurídico da Prefeitura de São Paulo, 1941, 599 p. - D. A. S. P. e T. C.

Revisão de tarifas do serviço telefónico, 1939.

Uruguar, Paulino José Soares de Sousa, visconde de - Ensaio sôbre o direito administrativo, Rio de Janeiro, Tipografia Nacional, 1862, 2 v.) - D. A. S. P., S. T. F., F. D. e T. C.

Estudos práticos sôbre a administração das províncias no Brasil, Rio de Janeiro, B. L. Garnier, 1865,2 v. - D. A. S. P., S. T. F. e T. C.

Tesouro Nacional, em quatro partes a saber: Da alta administração da Fazenda; Da administração central da Fazenda; Da administração da Favenda nas províncias; Da forma do proceso na administração da Fazenda, Rio de Janeiro, Tipografia Nacional, 1869, 434 p. F. D.

VALADÃo, Alfredo - Dos rios públicos e particulares, Belo forizonte, 1904. -S. T. F.

Projeto de reforma do Tribunal de Contas.

Regime jurídico das águas e da indústria hidro-elétrica.

Vale, Numa P. do - Da responsabilidade dos estados, São Paulo, Tipografia Jacob Zlotopolsky, 1925, 193 p. - D. A. S. P., S. D. M. J., S. T. F., F. D., M. F., T. A. e T. C.

Vasconcelos, José Matos de - Curso de administração geral, 1. a conferência : Direito administrativo e ciência da administração, Rio de Janeiro, Imprensa Militar, 1921, 483 p. - D. A. S. P.

Direito administrativo brasileiro, Rio de Janeiro, Tip. do Jornal do Cimércio, 1932, 110 p. - S. T. F. e T. C.

Direito administrativo, Rio de Janeiro, Imprensa Nacional, 1937, 2 v. - D. A. S. P., T. C., M. F. E T. A.

Vrrga CABral, Prudêncio Giraldo $T$. Tavares da - Direito administrativo brasileiro, Rio de Janeiro, Tip. Laemmert, 1859, 659 p. - S. T. F. e T. C. 
Vuava, Paulo Domingues Do estatuto dos funcionários públicos, Rio de Janeiro, Jacinto Ribeiro dos Santos, $1915,200 \mathrm{p}-$ D. A. S. P. e S. T. F.

VIVEIRos dE CASTRo, Augusto Olímpio - Desapropriação (in Revista de Direito, v. XVIII, dezembro, 1910, p. 409).

Tratado de ciencia da administração e direito administrativo, $2 .^{\mathrm{a}} \mathrm{ed}$., Rio de Janeiro, Jacinto Ribeiro dos Santos, 1912, 756 p.

3. ${ }^{\mathrm{a}}$ ed, 1914,864 p. - D. A. S. P., S. T. F., I. A. P. I. e M. F.

VIVÁquA, Atílio - A nova política do subsolo e o regime legal das minas, Rio de Janeiro, Editôra Pan-Americana, 1942, 637 p. - S.T.F.e M. F.

Whitaker, F. - Desapropriação, 2.a ed, São Paulo, 1926. - S. T. F. 